\title{
Evaluation of a training programme on the use of behavioural change techniques in childhood obesity management
}

\author{
L. Stewart \\ The Children's Weight Clinic, 8 Seaforth Drive, Edinburgh EH4 2BT, UK
}

The delivery of childhood weight management programmes has risen up the political agenda in the UK. The SIGN $115^{(1)}$ and NICE $43^{(2)}$ guidelines recommended that childhood weight management programmes incorporate behavioural change techniques. The present study aimed at assessing and evaluating changes in participants' confidence in the practical use of behavioural change tools after a 2-d training in paediatric obesity and its management.

A semi-quantitative questionnaire was distributed among participants pre- and post-training and completed by trainees anonymously. A five-point Likert scale was used to assess confidence. Descriptive analysis of data was undertaken to quantify the self-reported changes in confidence and practice from pre- to post-training.

Twenty-one participants, all dietitians (nineteenth health, two health and education) undertook the training in 2010. Twenty completed both the pre- and post-questionnaires. The scope of experience in childhood obesity ranged from none (1/21) to greater than 3 years $(11 / 21)$. Table 1 shows the changes in the Likert scoring pre- and post-training. Prior to the training $0 \%(0 / 20)$ gave a confidence score of $4 / 5$ on the Likert scale for assessing the child's readiness to change, with $71.4 \%(15 / 21)$ giving a $4 / 5$ score after training. For enabling the child to set goals prior to training, $33.3 \%$ (7/20) gave a 4/5 score, after $81 \%$ (17/21). Confidence in enabling problem solving increased from $5 \%(1 / 20)$ with a $4 / 5$ score to post-training $76 \%(16 / 21)$. Confidence in establishing rapport increased from a pre-training level of $71.4 \%(15 / 20)$ at $4 / 5$ score to $100 \%$.

Table 1. Showing changes in confidence pre- and post-training

\begin{tabular}{|c|c|c|c|c|c|c|c|c|}
\hline \multirow[b]{2}{*}{ Likert score } & \multicolumn{2}{|c|}{ Assessing child's readiness to change } & \multicolumn{2}{|c|}{ Enabling child to goal set } & \multicolumn{2}{|c|}{ Enabling problem solving } & \multicolumn{2}{|c|}{ Establishing rapport } \\
\hline & Pre & Post & Pre & Post & Pre & Post & Pre & Post \\
\hline 1 & 2 & & 1 & & 2 & & 1 & \\
\hline 2 & 1 & & 2 & & 5 & & 2 & \\
\hline 3 & 18 & 6 & 11 & 4 & 13 & 5 & 3 & \\
\hline 4 & & 13 & 7 & 12 & 1 & 13 & 13 & 13 \\
\hline 5 & & 2 & & 5 & & 3 & 2 & 8 \\
\hline
\end{tabular}

This study highlighted the importance of ensuring training for professional staff involved in childhood obesity to ensure they have the appropriate tools and confidence to use them in engaging with obese children and their families.

1. Scottish Intercollegiate Guideline Network (SIGN) (2010) Obesity SIGN 115. Edinburgh.

2. NICE (2006) Obesity guidance on the prevention, identification, assessment and management of overweight and obesity in adults and children. NICE 43, London. 